\title{
Declining Performance of Silicone-Based Magnetorheological Elastomers after Accelerated Weathering
}

\author{
Wibowo Wibowo ${ }^{1}$, Bhre Wangsa Lenggana ${ }^{1}$, Ubaidillah Ubaidillah ${ }^{1, *} \mathbb{( D}$, Dody Ariawan ${ }^{1}(\mathbb{D}$, \\ Fitrian Imaduddin ${ }^{1}{ }^{1}$, Saiful Amri Mazlan ${ }^{2, *}$ and Seung-Bok Choi ${ }^{3,4, *}$
}

1 Mechanical Engineering Department, Faculty of Engineering, Universitas Sebelas Maret, Surakarta 57126, Indonesia; wibowo69@staff.uns.ac.id (W.W.); bhrewangsa20@gmail.com (B.W.L.); dodyariawan@staff.uns.ac.id (D.A.); fitrian@staff.uns.ac.id (F.I.)

2 Engineering Materials and Structures (eMast) iKohza, Malaysia-Japan International Institute of Technology (MJIIT), Universiti Teknologi Malaysia, Jalan Sultan Yahya Petra, Kuala Lumpur 54100, Malaysia

3 Department of Mechanical Engineering, The State University of New York, Korea (SUNY Korea), Incheon 21985, Korea

4 Department of Mechanical Engineering, Industrial University of Ho Chi Minh City, 12 Nguyen Van Bao Street, Vap District, Ho Chi Minh City 70000, Vietnam

* Correspondence: ubaidillah_ft@staff.uns.ac.id (U.U.); amri.kl@utm.my (S.A.M.); seungbok.choi@sunykorea.ac.kr (S.-B.C.)

Citation: Wibowo, W.; Lenggana, B.W.; Ubaidillah, U.; Ariawan, D.; Imaduddin, F.; Mazlan, S.A.; Choi, S.-B. Declining Performance of Silicone-Based Magnetorheological Elastomers after Accelerated Weathering. Materials 2021, 14, 6389. https://doi.org/10.3390/ma14216389

Academic Editor: Ramin Sedaghati

Received: 28 August 2021

Accepted: 18 October 2021

Published: 25 October 2021

Publisher's Note: MDPI stays neutral with regard to jurisdictional claims in published maps and institutional affiliations.

Copyright: (c) 2021 by the authors. Licensee MDPI, Basel, Switzerland. This article is an open access article distributed under the terms and conditions of the Creative Commons Attribution (CC BY) license (https:/ / creativecommons.org/licenses/by/ $4.0 /)$.

\begin{abstract}
Magnetorheological elastomers (MRE)-based products are usually located in an area directly exposed to sunlight and rain. However, there is no specific research on the behavior of MRE after accelerated weathering. Therefore, in this study, the changes to the chemical and rheological properties of both isotropic and anisotropic MRE after accelerated weathering were examined. Treated and untreated specimens were compared. MRE specimens with $40 \%$ by weight CIP were prepared with no current excitation and another sample was prepared with $1.5 \mathrm{~T}$ of magnetic flux density. Each specimen was treated in an accelerated weathering machine, Q-Sun Xe-1 Xenon Test Chamber, under a UV light exposure cycle and water spray. A material characterization was carried out using FTIR and a rheometer to determine the changes to the chemical and rheological properties. The morphological analysis results showed that after the weather treatment, the surface was rough and more cavities occurred. The rheometer test results showed a significant decrease in the storage modulus of each treated MRE specimen, unlike the untreated MRE specimens. The decrease in the storage modulus value with currents of $0,1,2$, and 3 Amperes was $66.67 \%, 78.9 \%, 85.2 \%$, and $80.5 \%$, respectively. Meanwhile, FTIR testing showed a change in the wave peak between the untreated and treated MRE specimens. Thermogravimetric analysis (TGA) also showed a decrease in MRE weight for each specimen. However, for both treated and untreated MRE specimens, the decrease in TGA was not significantly different. In all the tests carried out on the MRE samples, weather acceleration treatment caused significant changes. This is an important consideration for developers who choose silicone as the MRE matrix.
\end{abstract}

Keywords: magnetorheological; elastomer; magnetorheological elastomer; MRE; weather; accelerated; rubber; composite; rheological

\section{Introduction}

The development of rubber products has progressed rapidly. One of these developments is engineered rubber products, widely applied in various engineering fields. These products have excellent physical properties and durability, as well as the ability to deform. Rubber composites are one type of engineered rubber product widely applied in various fields such as marine [1], structural, automotive [2,3], and aerospace [4]. Several studies have shown that rubber composite products have greater environmental sensitivity compared to metal structures, especially in their application in the space sector. However, 
engineered rubber products, such as composites, are highly susceptible to the conditions of ambient temperature, humidity, heat, and chemicals they encounter. In general, the aging of rubber composites is caused by chemicals [5,6], thermal effects, heat $[7,8]$, oxidative destruction [9-11], and moisture [12]. Thus, the weather conditions in which the applications of rubber composite products occur can negatively affect their physical properties [13]. A particular issue is the degradation or aging stability of rubber composites. The aging phenomenon is an irreversible process that can significantly change their physical, mechanical, and rheological properties [13,14]. The change in interface adhesion in reinforcement and rubber causes hardening of the rubber composite, resulting in brittleness and a reduced lifetime.

In addition to general rubber products applied in the automotive sector, such as tires or other specialty rubber products, rubber composites in the form of sensors and magnetic and electromagnetic field protection materials have been proposed in recent decades. For this kind of usage, a new type of rubber composite with a nonconventional filler as an integrated active component is required [15]. One way to achieve this purpose is to prepare a new material with a specific filler such as magnetic particles. Magnetorheological elastomers (MRE) are a new generation of materials widely explored for their excellent flexibility, ease of formation, and sensitivity to magnetic fields $[15,16]$. However, despite the capabilities of MRE as a composite material, it remains highly vulnerable to damage due to the influence of the surrounding environment [17]. MRE aging affects the mechanical properties and durability of MRE, resulting in damage. One example is the study conducted by Kruzelak et al. [10], which showed an increase in the crosslink density of magnetic composites due to the formation of rubber chain oxidation during oxidative aging. They observed that the mechanical properties associated with the rubber magnetic composites' modulus and hardness increased compared to the samples without aging. Masbowski et al studied thermo-oxidative aging in MRE samples. [18] MRE samples with carbonyl iron particles (CIP) and carbon black fillers were treated by being placed in a drying oven at $70{ }^{\circ} \mathrm{C}$ for two weeks [19]. The mechanical properties of the MRE slightly deteriorated due to the oxidative aging process. Other researchers drew similar conclusions; tensile strength, tear strength, and hardness decreased significantly up to $72 \%$ with the increase in temperature and aging time. This was due to the molecular configuration changes accompanied by hydrothermal deterioration that caused microgaps to develop between the matrix and the fill/matrix interface.

Treatment related to MRE degradation has been conducted in several forms, such as thermal treatment, but some of these treatments could not represent weather behavior. Based on previous research, rubber composite/MRE studies have been limited to specific rubber matrices and mainly focused on mechanical properties and crosslinking [20]. One study involved the use of RTV silicone rubber as a massive MRE matrix because of the ease of fabrication. However, intensive studies on the resistance of MRE based on RTV silicone to natural conditions such as sunlight, heat, and rainfall have not received attention [21,22]. Meanwhile, the application of rubber composite products such as MRE has been mainly in environments directly exposed to sunlight and rain $[23,24]$. Therefore, this article contributes to understanding the effect of weathering by using an accelerated weathering machine on MRE specimens based on silicone RTV, both isotropic and anisotropic types. New findings were realized by examining the rheological properties and material deterioration under infrared testing. The main finding could be important for researchers or designers in deciding whether to use silicone as the MRE matrix.

\section{Materials and Methods}

\subsection{Samples Preparation}

In this study, MRE samples were prepared based on silicone RTV consisting of $40 \mathrm{wt} . \%$ CIPs and silicone RTV as a suspending medium. The preparation of the MRS samples is shown in Figure 1. 


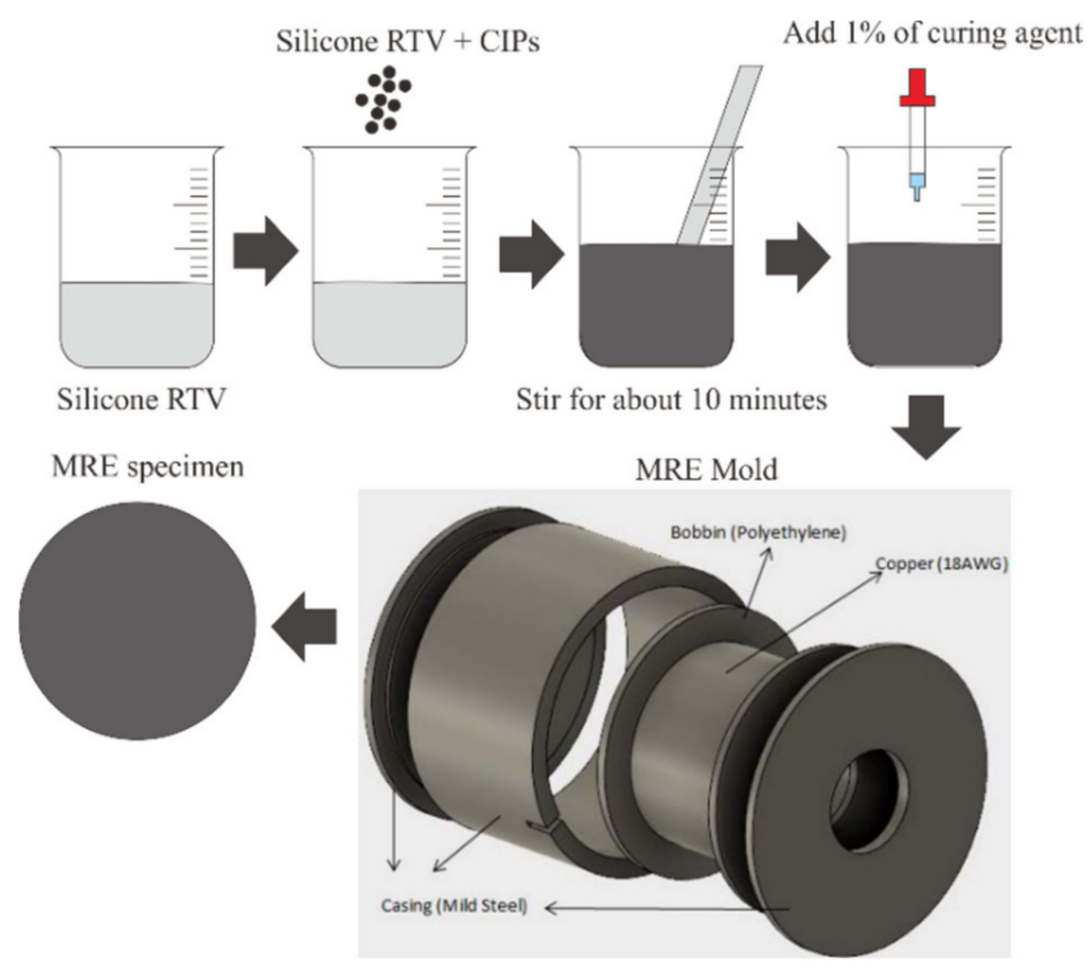

Figure 1. MRE sample preparation.

Both isotropic and anisotropic types were made using the same equipment. For the anisotropic MRE samples, the mixed silicone RTV and CIP were cured under the influence of a magnetic flux density of $0.5 \mathrm{~T}$. This flux magnetic value was obtained by applying 1 ADC current. To determine the value, the flux density was measured using a gauss meter before the mold was filled with the mixture. The MRE sample was stirred conventionally for about $10 \mathrm{~min}$ at room temperature $\left(25^{\circ} \mathrm{C}\right)$ until it was visually homogenous. Then, a $1 \%$ curing agent, NS $625 \mathrm{~B}$ (Nippon Steel) was added to the stirred MRE sample before it was poured into the mold. To remove small bubbles, the mixtures were placed in a vacuum chamber for degassing purposes [15].

\subsection{Sample Characterization}

The test method followed the standard test method for rubber deterioration using an artificial weathering apparatus. This process was conducted in an accelerated weathering machine Q-SUN Xe-1 Xenon Test Chamber and ASTM D750. The Q-SUN Xe-1 Xenon Test Chamber is a tabletop chamber that reproduces the damage caused by full-spectrum UV light using a single xenon arc lamp with optional water spray and chiller which is manufactured at Q-Lab Florida, FL, USA.

As stated in ASTM D750, the specimens should have a maximum thickness of $0.75 \mathrm{~mm}$ and a minimum thickness of $0.60 \mathrm{~mm}$ (0.025 in); however, the width of the specimens is not critical. Along with the ASTM standard thickness, samples with a thickness of $1 \mathrm{~mm}$ were also treated based on the needs of the rheology testing, which requires a minimum thickness of $1 \mathrm{~mm}$. The default exposure was $102 \mathrm{~min}$ light only, followed by $18 \mathrm{~min}$ light plus either water spray on the front surface or immersion in water. The black panel temperature (BPT) for this test was $63^{\circ} \mathrm{C}$ during the dry period of exposure to light, with a relative humidity of $60 \%$ during this exposure. The irradiance level was controlled at $340 \mathrm{~nm}$. This test ran an $8 \mathrm{~h}$ cycle of UV exposure in an uninsulated black panel with a temperature of $60^{\circ} \mathrm{C}$, followed by a condensation cycle of $4 \mathrm{~h}$ in the dark, with wetting and an uninsulated black panel temperature of $50{ }^{\circ} \mathrm{C}$. The samples obtained were MRE samples with and without treatment, for both isotropic and anisotropic types. 
The prepared samples were then characterized by several tests such as morphology, rheology, and Fourier transform infrared (FTIR). To examine the surface of the treated and untreated samples, image capture for micrograph analysis was performed at ten magnification (M10) using a Euromex microscope model "F" range, Holland by Euromex Microscopen BV, Arnhem, Netherlands. This enlargement was clear enough to identify differences between the treated and untreated samples. Micrograph analysis was carried out on the surface of the samples, since during the treatment with an accelerated weathering machine, the sample surfaces were directly exposed to both UV rays and water. The MRE samples for rheometer testing were cut to a diameter of $20 \mathrm{~mm}$. This test was performed using a Rheometer MCR 302 Anton Paar Companies machine. The amount of the MRE sample between the CIPs and silicone rubber medium in this study was based on the weight ratio. The two ingredients were stirred manually at room temperature for $10 \mathrm{~min}$ until homogeneous. After homogeneity was reached, each sample was poured on the mold and levelled before clotting. The samples were tested with a rheometer at $25^{\circ} \mathrm{C}$. In addition to rheometer testing, FTIR testing was carried out on samples to determine any changes or differences in chemical compounds between the untreated and treated MRE samples. The MRE samples to be tested by FTIR were crushed until smooth using a mortar stamper. FTIR testing used a Shimadzu Prestige 21 IR, Japan, with a wavelength specification of $12500-240 \mathrm{~cm}^{-1}$ and an accuracy level of $\pm 0.125 \mathrm{~cm}^{-1}$. The spectrum resolution on this machine ranged from 0.5 to $2 \mathrm{~cm}^{-1}$.

\section{Results and Discussion}

\subsection{Micrograph Analysis}

Micrograph studies of the surfaces of the treated and untreated MRE samples were essential to analyze the degree of degradation. Figure 2a shows an untreated micrograph sample, and Figure $2 \mathrm{~b}$ shows a micrograph sample with weather treatment using an accelerated weathering machine. The effects of surface degradation are evident in these images $[25,26]$, with the results showing a clear difference.

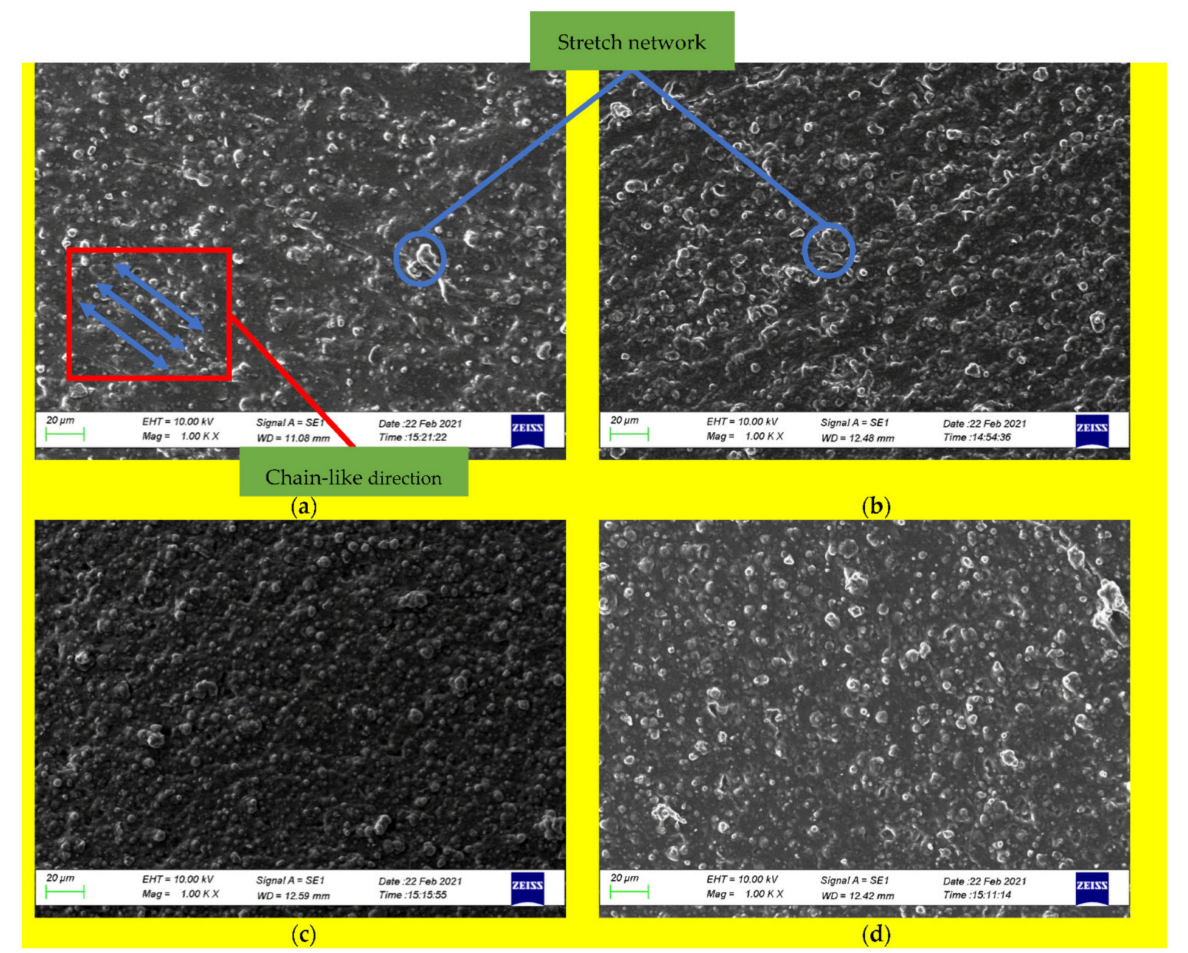

Figure 2. SEM results of cross-sectional MRE sample slices; (a) anisotropic untreated; (b) anisotropic treated; (c) isotropic untreated; $(\mathbf{d})$ isotropic treated. 
After treatment, the MRE sample surface was more irregular than that of the virgin sample. The change in the roughness of the treated samples was clear, even using a regular microscope. Surface defects were possibly due to the evolution of low molecular degradation products, which allow the penetration of water and possible decomposing agents (e.g., enzymes) into the bulk of the polymer and facilitate further environmental degradation. This phenomenon was also found by other researchers who tested MRE with thermal aging [27].

Micrograph analysis was performed using an SEM (Scanning Electron Microscope) ZEISS EVO 10 by Carl Zeiss Company, Jena, Germany. The analysis was carried out with a magnification of 1000 times for both sample variations. SEM testing in this study aimed to see a clearer description of the elastomeric structure with a larger magnification scale, so that a comparison could be completed between the MRE samples with and without weather treatment. The test was carried out on the surface of the sample and the section of the sample slice, so that the chain structure formed on the MRE could be seen. The samples compared were isotropic and anisotropic MRE samples, treated and not weather treated. The results are shown in Figure $2 a, b$; the anisotropic MRE sample without weather treatment had a more neatly arranged structure and its appearance was a chain arrangement line that was cured with $1 \mathrm{~A}$ input. Weather-treated anisotropic MRE samples showed a more irregular (random) structure. A stretch network was seen more in the MRE samples with weather treatment, due to the samples being exposed to UV light alternately and sprayed with water continuously during the treatment process. The existence of the stretch network and the random arrangement of the chains caused the rheological properties of MRE to decrease significantly, as evidenced by the graph of the rheological test results.

For the MRE sample without weather treatment, the chain link structure of the sample slices was still clearly formed; while in the weather treated sample, the path was not visible because it appeared more random. This caused a decrease in the properties in the sample, according to the results of the graph. The weather treatment was carried out only on the surface of the sample, but the impact that occurred to the center of the sample was shown through the slices of the sample being tested. The results of the SEM test are shown in Figure 2. Data were collected using the same method and magnification. In addition, the number and size of stretch networks on the surface were also significantly different. At the same magnification, the surface of the anisotropic MRE samples with weather treatment was faded and grayer. This is, of course, a result of the UV rays being alternately exposed to the sample with constant water spray. In terms of color changes between the surface and the middle/inside of the MRE samples analyzed by SEM, the interior tended to remain the same color with or without treatment, since the inside was not exposed to the UV light. Meanwhile, judging from the number and size of the resulting stretch networks, the MRE samples with the weather treatment were more numerous and evenly distributed over all parts of the surface with a smaller size than the untreated MRE samples. However, in both of them, the chain structure path was not visible as clearly seen in the sliced MRE samples. The structure of both were irregular.

Isotropic MRE samples were also tested with the same method and magnification. Overall, the SEM results showed similarities, as presented in Figure 2c,d. The samples with the weathering treatment had more bubbles. This occurred in all the tests, both on the surface and on the slices. However, in contrast to the anisotropic MRE sample where a chain-like structure was formed, in this sample, structural bonds such as chains were not formed, because the manufacturing process did not use a magnetic field. This is shown in Figure 2c,d, with the 1000 times magnified samples of MRE slices showing a very random distribution of bonds. The difference in the number and size of bubbles in this isotropic MRE sample was not significant compared with the previous anisotropic sample. This is evidenced by the decrease in storage modulus, which is not significant, as shown in Figure 3c. The results are different from the decrease that occured in the anisotropic MRE samples. 


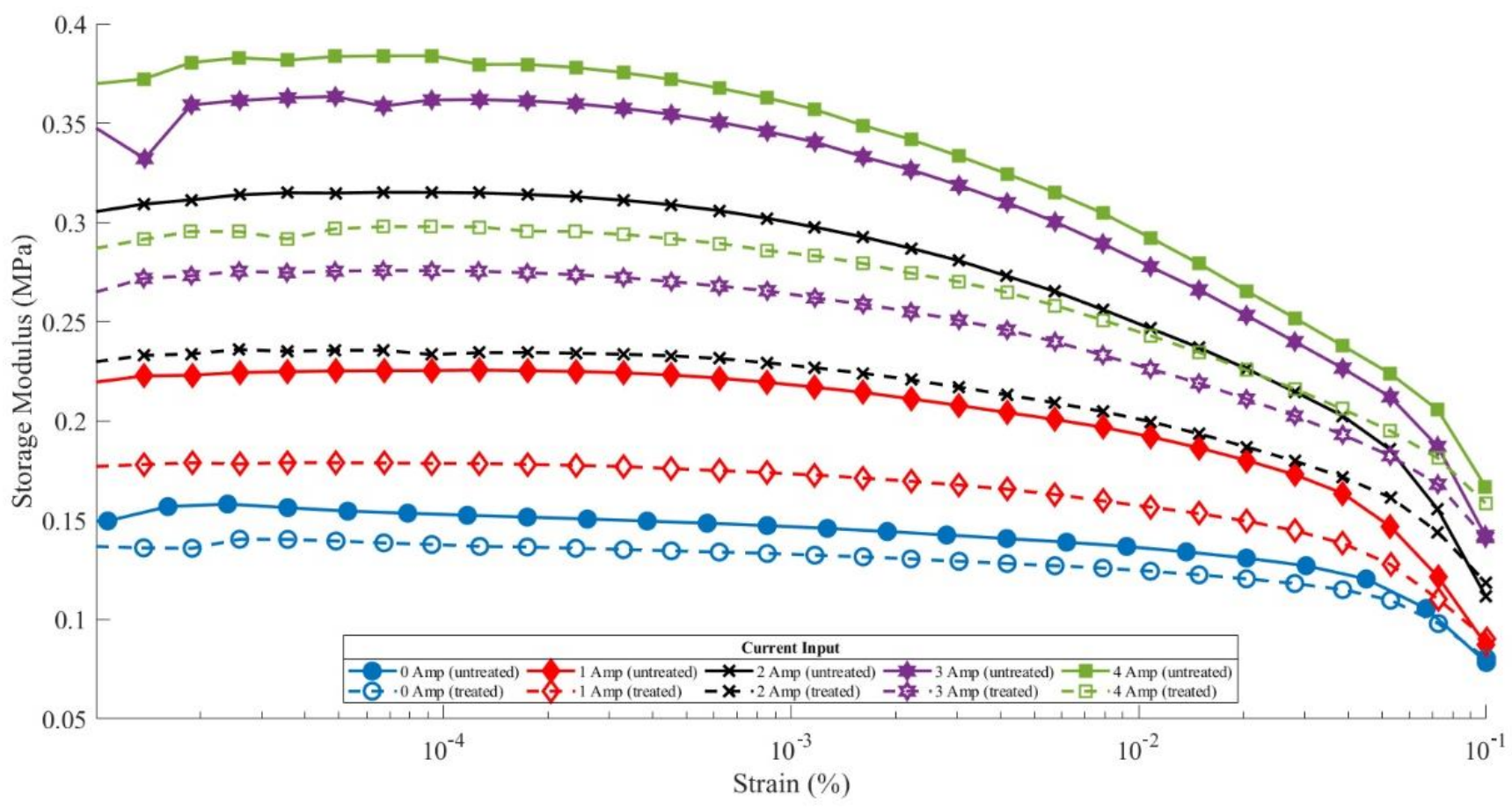

(a)

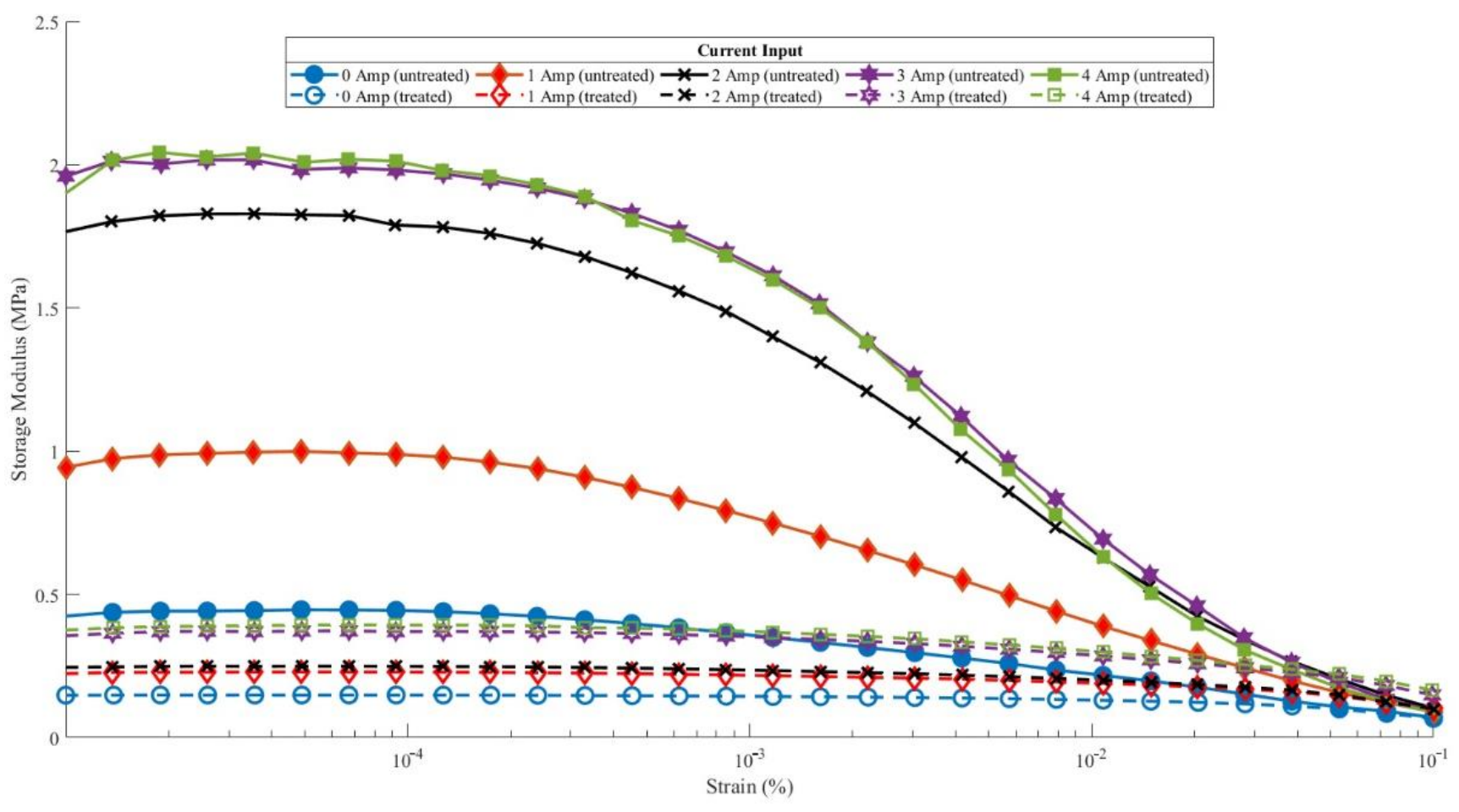

(b)

Figure 3. Cont. 


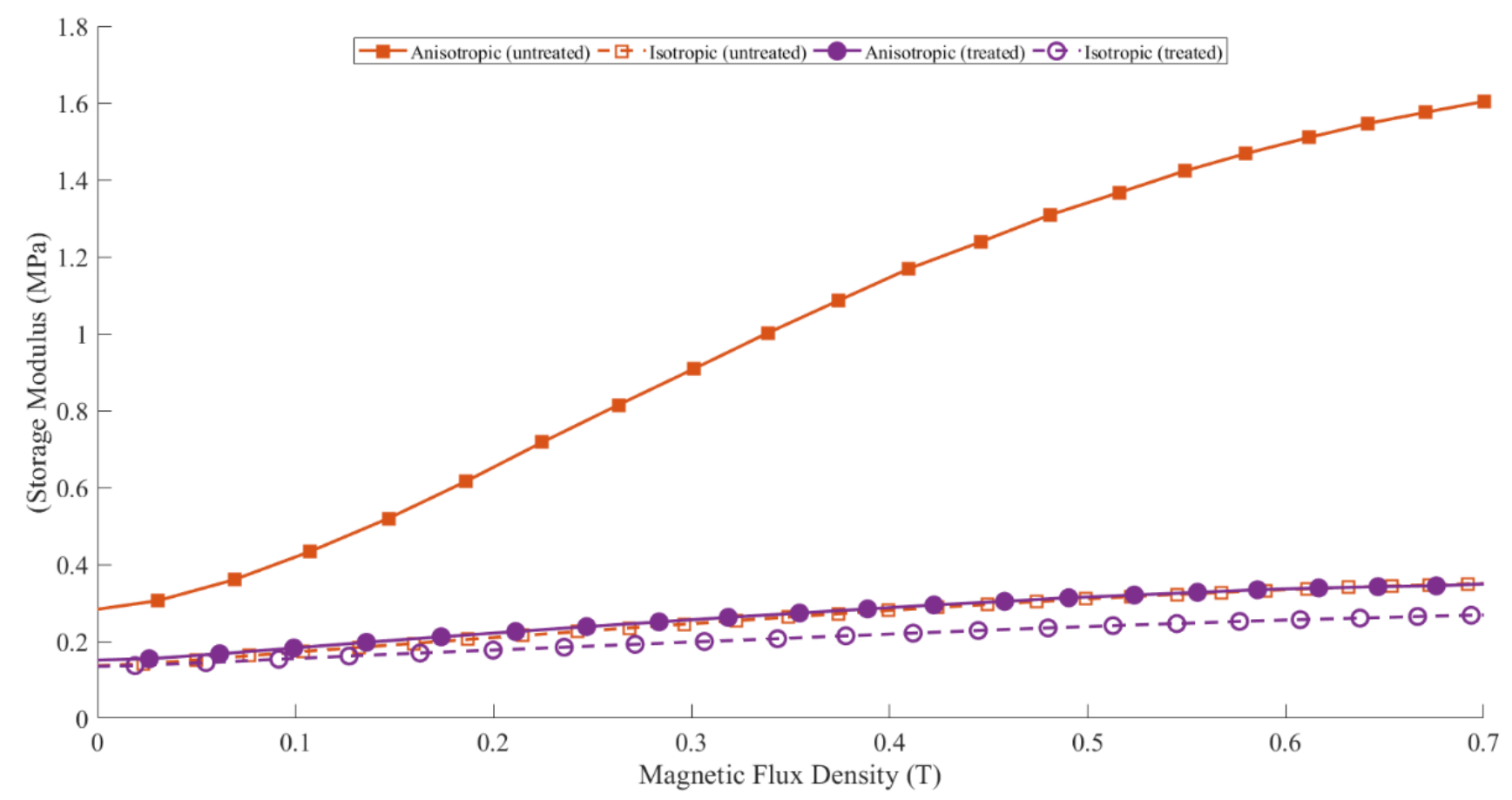

(c)

Figure 3. Results of rheometer characterization (a) isotropic; (b) anisotropic; and (c) comparison of anisotropic and isotropic under ramp frequency test.

\subsection{Rheological Properties}

The storage modulus of all samples with and without weather treatment is depicted in Figure 3. As shown in Figure 3, the storage modulus at each MRE sample flow strength, both anisotropic and isotropic, decreased. In Figure 3c, the treated anisotropic MRE sample showed a storage modulus value that was almost identical to the storage modulus of an isotropic MRE sample without treatment. This indicates that weather treatment can damage the particle chain arrangement that occurs in anisotropic MRE samples [28-31]. The breakdown of the particle chain arrangement occurred in all anisotropic MRE specimens and brought all storage modulus values close to the storage modulus of the isotropic MRE samples.

Figure 3 shows that the anisotropic MRE sample without treatment had a peak storage modulus value of $1.9 \mathrm{MPa}$ at the current input of $4 \mathrm{~A}$. Simultaneously, the peak storage modulus value of the anisotropic MRE sample treated at the same current input (4 A) was $0.37 \mathrm{MPa}$.

This shows that the storage modulus value decreased by $80.5 \%$ due to the influence of weather (exposure to UV rays and water spraying), which was carried out in a test cycle. A decrease in the storage modulus value occurred in all current inputs in the rheometer test. The decrease in the storage modulus value with currents of $0,1,2$, and 3 Amperes was $66.67 \%, 78.9 \%, 85.2 \%$, and $80.5 \%$, respectively. This reduction was highly significant compared to that of the isotropic MRE sample, as shown in Figure 4. The anisotropic MRE sample had a high storage modulus value due to the arrangement of the particle chains formed with the input of an electric current during the curing process of creating the samples. 


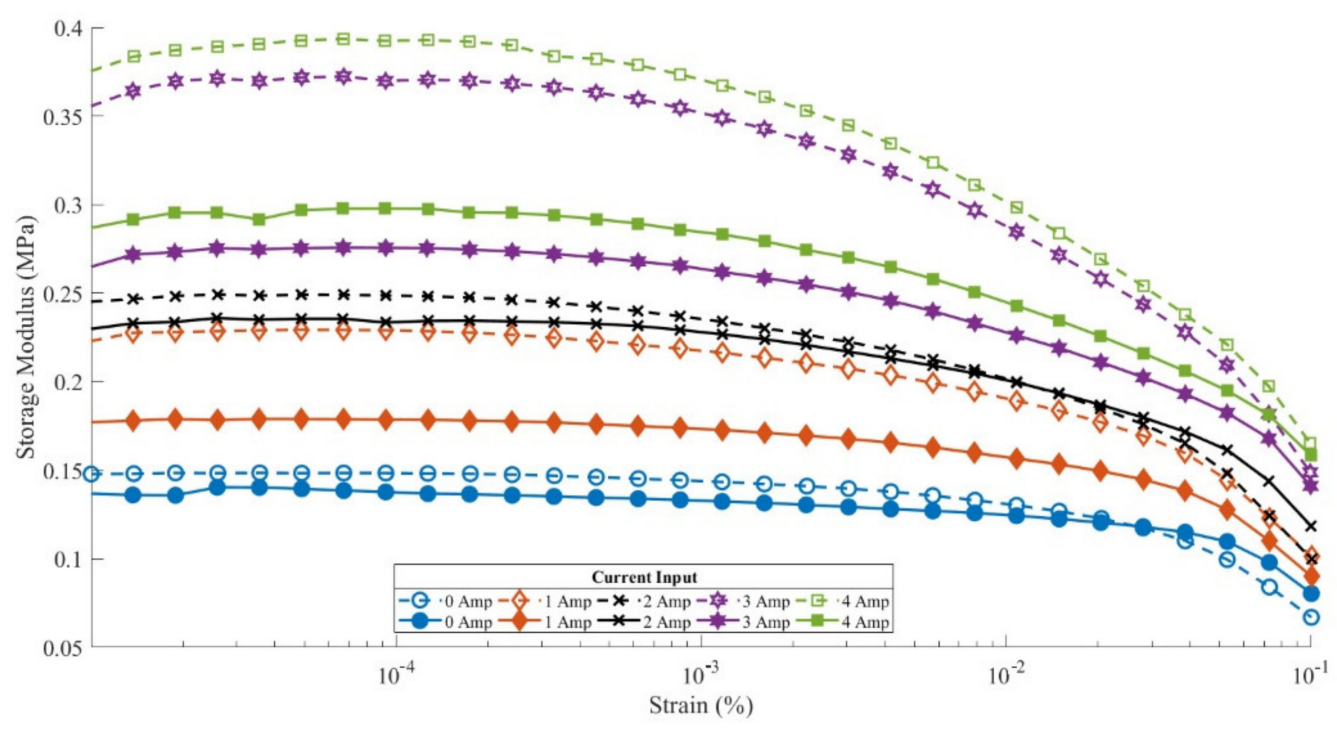

Figure 4. Results of MRE sample rheometer test for anisotropic treated and isotropic untreated.

A ramp frequency or frequency sweep is a particularly useful test as it enables the viscoelastic properties of a sample to be determined as a function of timescale. The storage modulus can be used as a measure of the elastic component of the sample and similarly, the loss modulus-the viscous component of the sample. Whichever modulus is dominant at a particular frequency will indicate whether the fully structured material appears elastic or viscous, in a process on a similar time scale. The mechanical response of most dispersion coatings is viscoelastic, since the presence of suspended solids, high additive concentrations, colloidal thickeners, etc., will induce a structure upon the bulk phase.

Figure 4 shows the results of the isotropic MRE sample rheometer test on the treated and untreated MREs. The peak value of the storage modulus of the isotropic MRE without treatment only reached $0.37 \mathrm{MPa}$ at $4 \mathrm{~A}$ current input when tested. This value was almost the same as the storage modulus value of the anisotropic MRE treated sample. Meanwhile, the isotropic treated MRE sample's storage modulus value only reached a peak value of $0.28 \mathrm{MPa}$. This shows that the decrease in the value of the isotropic MRE sample's storage modulus at the $4 \mathrm{~A}$ current input was $24.3 \%$. This reduction value is not comparable to that of the anisotropic MRE sample, which achieved a mean reduction of up to $78.3 \%$. This occurred because the arrangement of the particle chains in the isotropic MRE sample was irregular, so the weather treatment given to the sample did not damage the arrangement but only degraded its rheological properties [32,33].

\subsection{FTIR Testing}

FTIR testing carried out on the test samples produced data in the form of a graph of wavelength $\left(\mathrm{cm}^{-1}\right)$ vs. \% transmission, as shown in Figure 5. The graph shows the existence of a transmission in the form of a peak to be interpreted. This interpretation shows the bonds formed in the test specimens after weathering treatment and in the specimens without treatment.

The functional groups of the sample were elucidated with Fourier Transformation Infrared (FTIR) spectroscopy and the result is presented in Figure 5. The peak at around 2958 and $1260 \mathrm{~cm}^{-1}$ was attributed to the stretching and bending vibration of $\mathrm{Si}-\left(\mathrm{CH}_{3}\right)_{2}$ [34]. Meanwhile, two absorption bands at 1090 and $1018 \mathrm{~cm}^{-1}$ were assigned to the characteristics of silicone rubber for $-\mathrm{Si}-\mathrm{CH}_{2}-\mathrm{CH}_{3}$ [35]. Meanwhile, the peak around $800 \mathrm{~cm}^{-1}$ corresponded to silanol groups that could be deconvoluted to three peaks, as illustrated in Figure 7, using Equation (1). The peaks at 795, 800, and 810 were $\mathrm{Si}-\mathrm{O}-\mathrm{Si}$, terminal $\mathrm{Si}-\mathrm{OH}$ and unbonded $\mathrm{Si}-\mathrm{O}$, respectively [36]. FTIR deconvolution is a method of estimating quantitative interaction in a certain functional group [37]. The intensity of the bond formation in each specimen appeared to be the same. However, there was a difference at a 
wavelength of $3451 \mathrm{~cm}^{-1}$, as the untreated specimens showed the formation of a peak with a reasonably comprehensive intensity, compared to the treated specimens, both anisotropic and isotropic. There was almost no peak appearance in the treated specimens, as shown in the results of the treated anisotropic specimens. This indicates that the $\mathrm{OH}$ group formed before the treated specimen faded due to the influence of UV exposure.

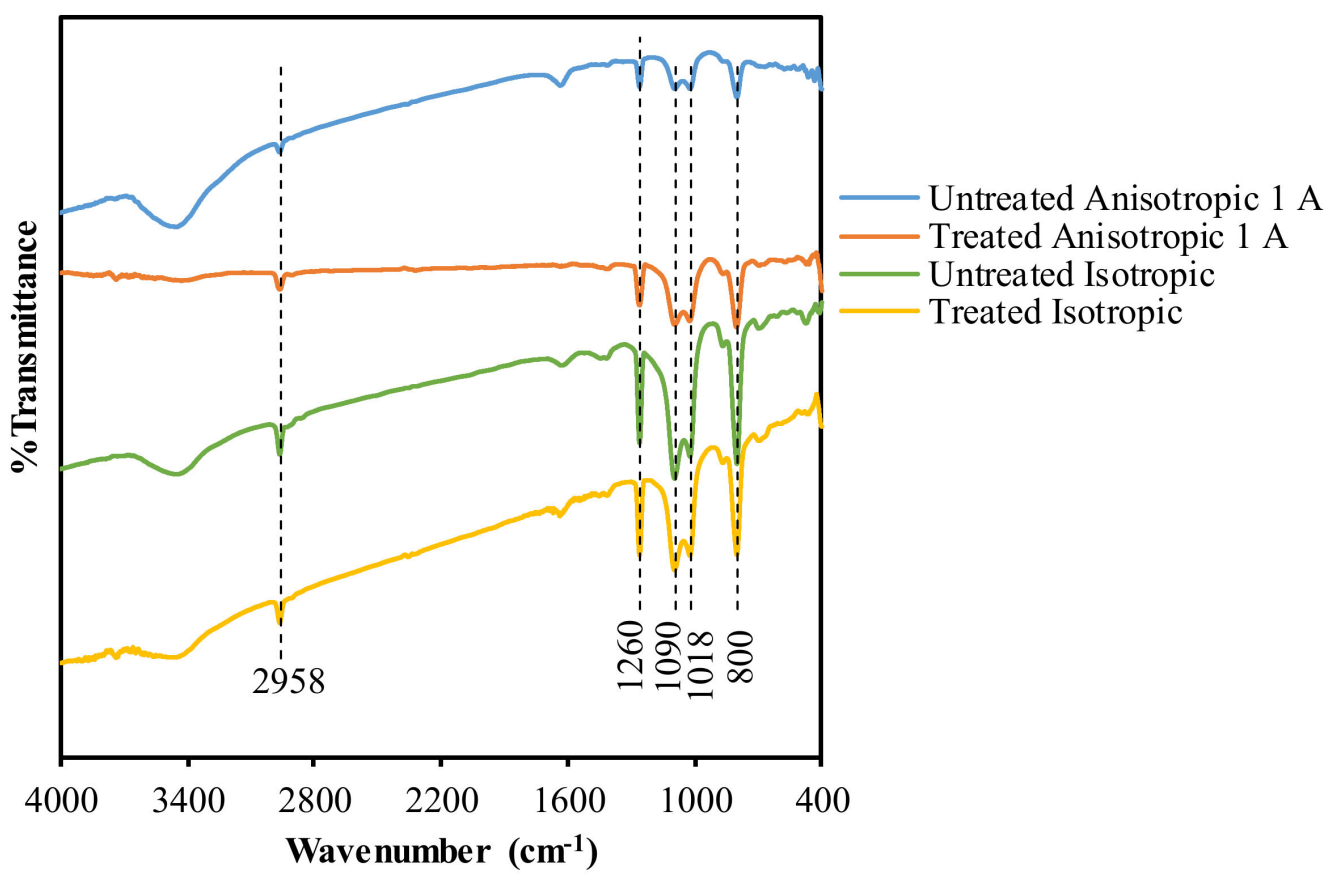

Figure 5. FTIR results.

Of all the tested MRE specimens, no absorption was found at $560-570 \mathrm{~cm}^{-1}$, which should be the iron oxide or $\mathrm{Fe}-\mathrm{O}$ absorption region [38,39]. This behavior is called a Binder bond [40]. The binder bond occurs due to the neutralization of the carbonyl charge so that it cannot interact with infrared wavelengths. Meanwhile, using the deconvolution method, the Gaussian peak area is presented in Figure 6. The peak area of the treated sample showed decreasing trends, possibly due to the iron filler detaching from the silicone rubber. The Figure $7 \mathrm{a}-\mathrm{d}$ show the consistency of the deconvolution results at the three peaks. Finally, the iron carbonyl as a filler had no chemical interaction with the silicone rubber because the rubber coated the iron filler, as reported in other research [41].

$$
\mathrm{Y}=\frac{A}{w} \sqrt{\frac{4 \ln 2}{\pi}} \frac{1}{4 \ln 2} \frac{(x-x c)^{2}}{w^{2}}
$$

where $A$ and $w$ are the area and full width of the graph, respectively, with $x c$ as the center. 


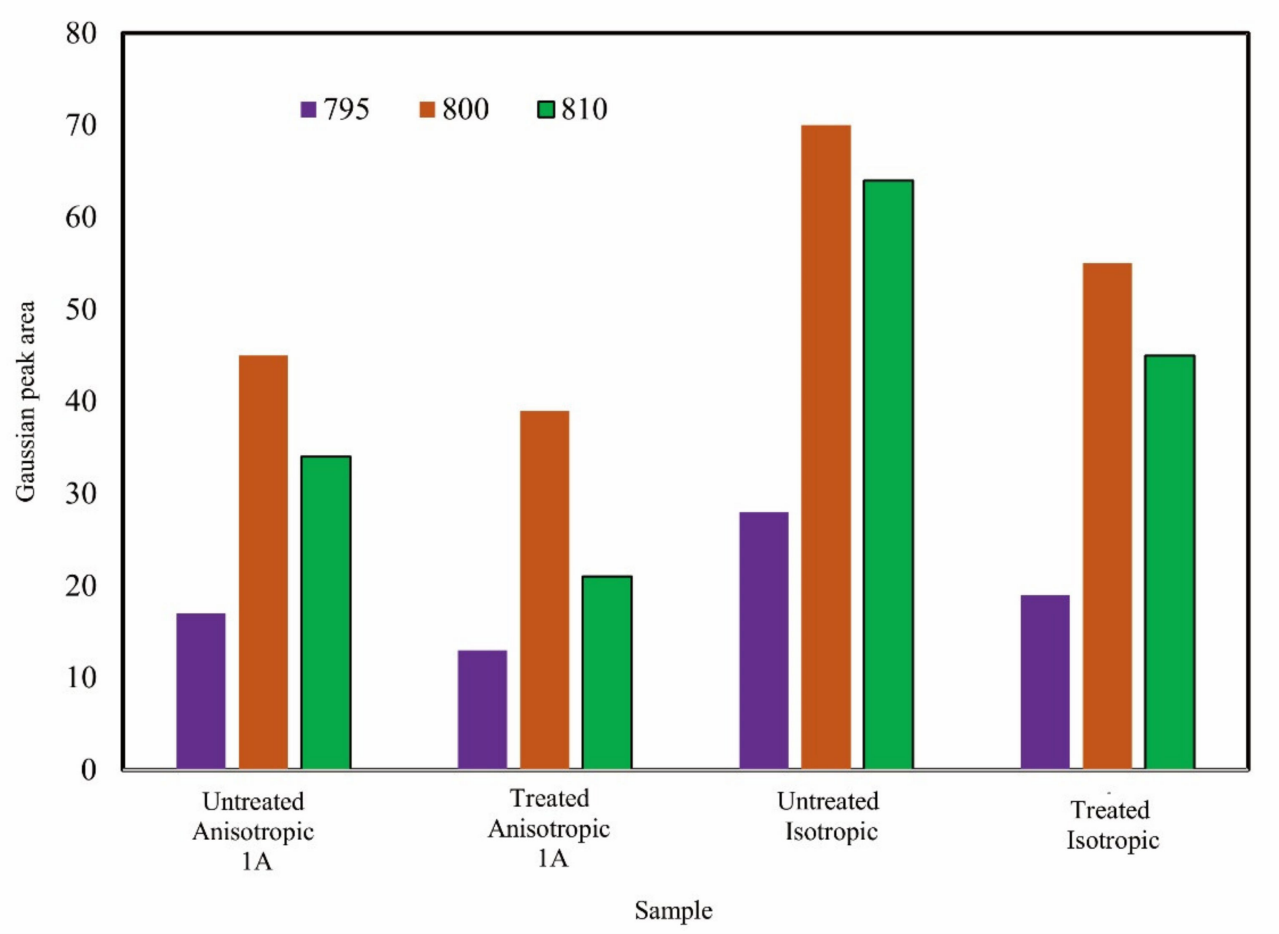

Figure 6. Gaussian peak area at $800 \mathrm{~cm}^{-1}$.
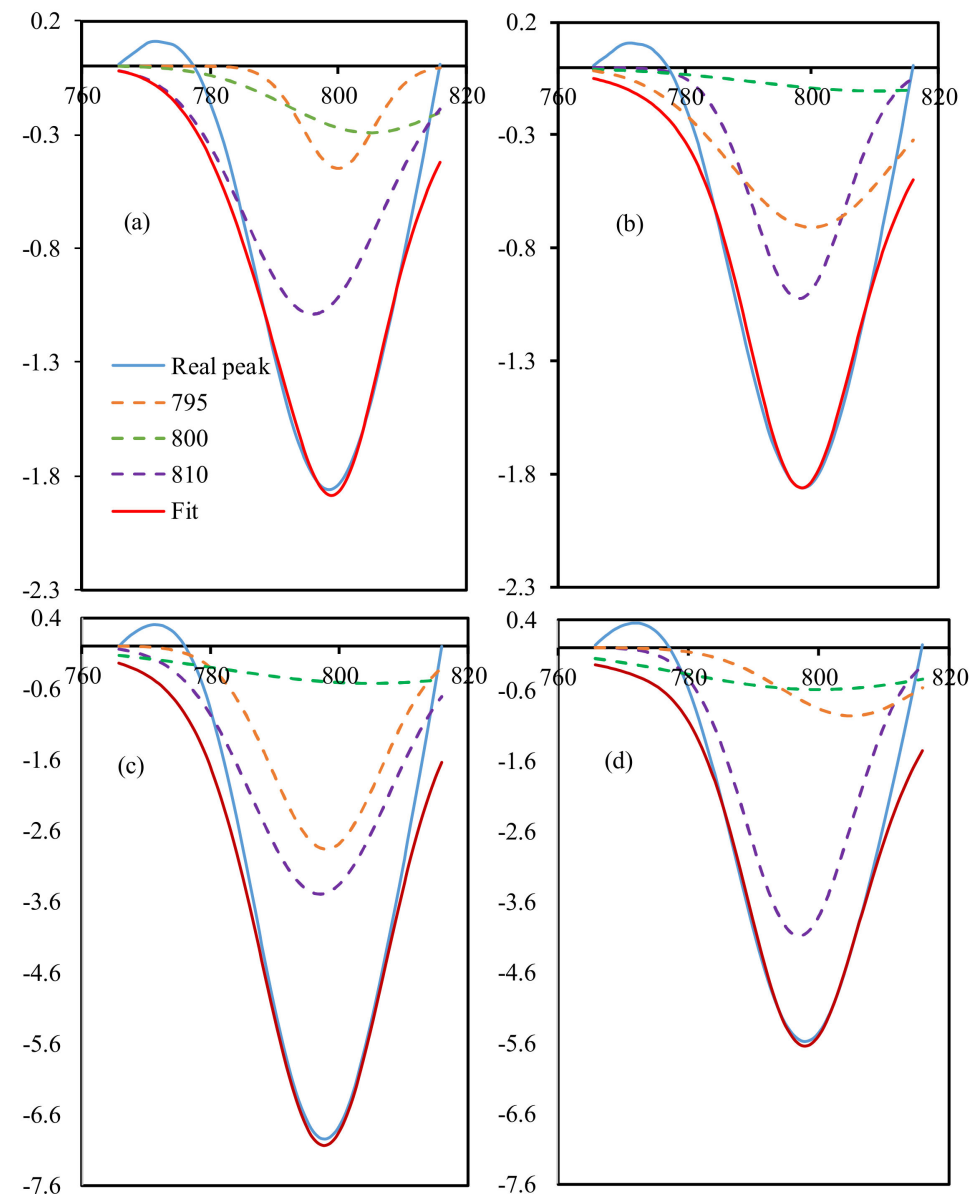

Figure 7. FTIR deconvolution of peak $800 \mathrm{~cm}^{-1}$. 


\subsection{Thermogravimetric Analysis (TGA)}

Thermogravimetric analysis was performed using a TGA Q50 by TA Instruments, New Castle, DE 19720, USA. The magnetorheological elastomers test sample had a mass of about $5-10 \mathrm{mg}$ with a temperature variation of 30 to $600{ }^{\circ} \mathrm{C}$ used. The test was carried out using a heating rate of $20^{\circ} \mathrm{C} / \mathrm{min}$ under a nitrogen gas environment (flow rate $100 \mathrm{~mL} / \mathrm{min}$ ). An expansion probe was equipped to measure the change in dimensions of the samples measuring $5 \times 5 \times 10 \mathrm{~mm}$. Data were collected at a heating rate of $10^{\circ} \mathrm{C} / \mathrm{min}$ from an ambient temperature of $150{ }^{\circ} \mathrm{C}$ while the initial load was set at $0.05 \mathrm{~N}$. Figure 8 shows the thermogravimetric results of several variations of sampled magnetorheological elastomers. The tests were carried out on the isotropic and anisotropic samples and the results were also compared between samples with weather treatment and without.

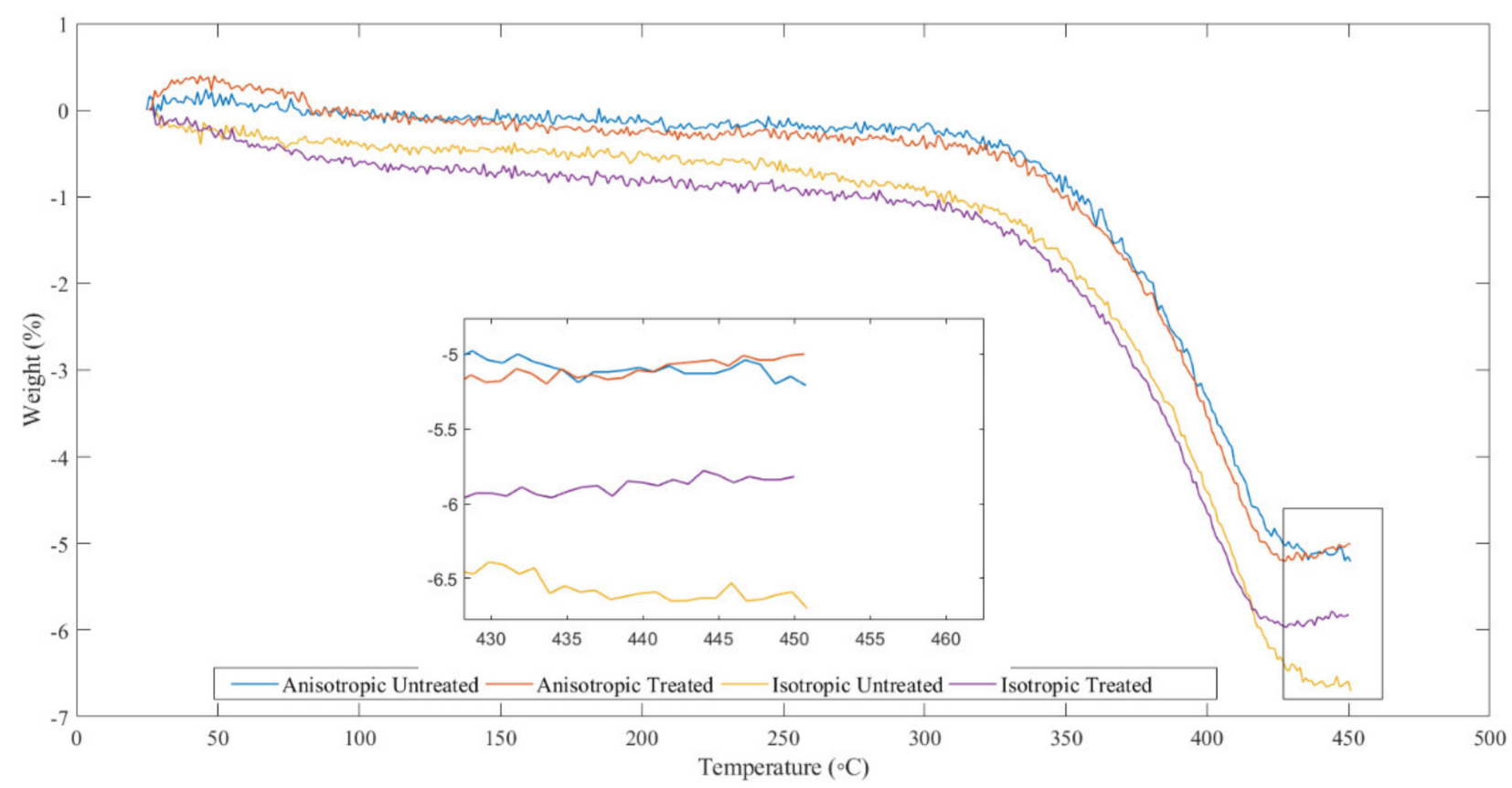

Figure 8. Thermogravimetric curves of MRE specimens.

Boczkowska et al. (2006) carried out a similar analysis using MRE samples. Thermogravimetric testing is one part of thermal analysis, which determines the level of stability of a material against external temperatures. In addition, the oxidative mass reduction, and its decomposition performance can also be determined through this analysis. Boczkowska et al. (2006) reported that at a temperature of around $600{ }^{\circ} \mathrm{C}$ additional iron particles in the elastomeric matrix will increase stability [42], indicated by the presence of lower oxidative mass loss. The thermal conductivity of the MRE sample was said to be improved due to the higher magnetic particles in the MRE sample, which increased its thermal conductivity. This happens because the distance between the particles is closer. Therefore, the flow of heat energy distributed throughout the sample will be greater if the MRE element has a higher number of iron particles.

In this study, the level of thermal stability of the MRE sample was evaluated using thermogravimetric analysis (TGA) Q50 by TA Instruments, New Castle, DE 19720, USA. In addition, the content of carbon black and ash compounds in the rubber used in the sample can be observed with this analysis. Figure 8 shows the TGA thermogram of the MRE samples. The rubber content of all samples completely decomposed. The initial temperature of the volatile compounds released in all samples was greater than $300{ }^{\circ} \mathrm{C}$. The volatile content of the samples was determined to be around $5.15-9.85 \%$. There was a mass loss of five percent (T5), increasing from $297.65^{\circ} \mathrm{C}$ to $421.65^{\circ} \mathrm{C}$ (anisotropic MRE with weather treatment). Mass loss in other samples, anisotropic MRE without treatment 
and isotropic with and without treatment, also increased at the same temperature. The last curve was maintained at the same weight until the next transformation. The content of carbon and CI particles changed at a temperature of more than $450{ }^{\circ} \mathrm{C}$. The weight reduction reflects the composition of the $\mathrm{CI}$ particles in the rubber and confirms the substance of the rubber. Lower CI particle content indicates a higher sample weight reduction. In this study, the weight loss of each sample at the end was $4.79,4.83,3.35$, and $4.03 \%$ for the anisotropic MRE samples without treatment, with treatment, isotropic without treatment, and with treatment, respectively. The remaining elements $\left(\right.$ at $\left.450{ }^{\circ} \mathrm{C}\right)$ as described in Table 1 were carbon black and CI particles. The test results showed that the thermal stability of the anisotropic MRE samples was greater than the isotropic MRE samples. This relates to the chain-like formation of particles in the anisotropic MRE samples. The residues of the MRE samples after the thermogravimetric test are shown in Table 1 below.

Table 1. Residual mass of MRE specimens.

\begin{tabular}{cc}
\hline Sample & Residue (\%) \\
\hline Anisotropic without treatment & 1.95 \\
Anisotropic with treatment & 2.25 \\
Isotropic without treatment & 0.05 \\
Isotropic with treatment & 0.6 \\
\hline
\end{tabular}

The tested MRE samples showed that the residue from the MRE samples with both isotropic and anisotropic weather acceleration treatments was greater than the samples without treatment. Wang et al. found a similar pattern with different compositions of carbonyl iron particles in the MRE content and concluded that the greater thermal stability was shown by the MRE material, which had a higher carbonyl iron particle content $[43,44]$. The residual results shown in Table 1 are comparable. The residue produced by the MRE sample without weather treatment showed a significant difference from the treated sample, because the carbonyl iron particle content in the treated MRE samples had decreased or even been damaged, as previously described in the micrograph analysis, where many cracks and discoloration were visible. The rheological results were also in accordance with the statement that accelerating weather treatment causes a decrease in the performance of the material.

\section{Conclusions}

Weather treated and untreated MRE samples were characterized. The results from the analysis micrograph showed a significant difference between the treated and untreated samples. The degradation of the treated samples was evident in their increased roughness and the particle arrangement's surface irregularity. The results of the characterization test using rheology showed a highly significant reduction in the storage modulus. This decrease was up to $80.5 \%$ and occurred due to the aging of the samples treated using an accelerated weathering machine with exposure to UV light and continuous and repeated spraying of water on the sample. This process occurred due to the breaking of the chain of particle bonds arranged in the MRE sample; hence, the MRE particle chain formed in the anisotropic sample was damaged and resembled an isotropic sample. In FTIR testing, each sample showed little difference, with the appearance of a peak at the same wavelength with almost the same intensity. Nevertheless, a distinguishing factor was that at a wavelength of $3451 \mathrm{~cm}^{-1}$, the intensity of the untreated samples in forming $\mathrm{OH}$ groups was very low. Even the untreated anisotropic sample barely formed a peak at that wavelength. Thus, based on the results of the MRE characterization after weather treatment, the aging of the samples was faster, so a decrease in sample performance occurred. Exposure to UV light and water spray greatly affected the performance of the MREs, which are widely applied in open environments. This decrease in performance was evident in the rheological characterization test, which showed that the storage modulus of the treated sample decreased significantly compared to the untreated samples. This 
represents a new challenge for ongoing research in the field of advanced materials and magnetorheological elastomers, in particular.

Author Contributions: Conceptualization, U.U. and S.-B.C.; methodology, B.W.L. and F.I.; validation, D.A. and S.A.M.; data curation, D.A.; writing—original draft preparation, W.W.; supervision, U.U. and S.-B.C. All authors have read and agreed to the published version of the manuscript.

Funding: This research was funded by Hibah disertasi doktor PNBP 2021.

Institutional Review Board Statement: Not applicable.

Informed Consent Statement: Not applicable.

Data Availability Statement: Data sharing is not applicable for this article.

Acknowledgments: The authors thank Hibah disertasi doktor PNBP 2021 for funding this research.

Conflicts of Interest: The authors declare no conflict of interest.

\section{References}

1. Bus, T.; Dale, M.L.; Reynolds, K.J.; Bastiaansen, C.W.M. Thermoplastic, rubber-like marine antifouling coatings with microstructures via mechanical embossing. Biofouling 2020, 36, 138-145. [CrossRef]

2. Louda, P. Applications of thin coatings in automotive industry. J. Achiev. Mater. Manuf. Eng. 2007, 24, 51-56. Available online: http://jamme.acmsse.h2.pl/papers_vol24_1/24105.pdf (accessed on 14 March 2021).

3. Fentahun, M.A.; Savas, M.A. Materials Used in automotive manufacture and material selection using Ashby charts. Int. J. Mater. Eng. 2018, 8, 40-54. [CrossRef]

4. Zhang, H.; Xu, G.; Chen, X.; Wang, R.; Shen, K. Effect of long-term laboratory aging on rheological properties and cracking resistance of polymer-modified asphalt binders at intermediate and low temperature range. Constr. Build. Mater. 2019, 226, 767-777. [CrossRef]

5. García-Moreno, I.; Caminero, M.; Rodríguez, G.P.; López-Cela, J. Effect of thermal Ageing on the impact and flexural damage behaviour of carbon fibre-reinforced epoxy laminates. Polymers 2019, 11, 80. [CrossRef] [PubMed]

6. Kashi, S.; De Souza, M.; Al-Assafi, S.; Varley, R. Understanding the effects of in-service temperature and functional fluid on the ageing of silicone rubber. Polymers 2019, 11, 388. [CrossRef]

7. Muslov, S.A.; Polyakov, D.I.; Lotkov, A.I.; Stepanov, A.G.; Arutyunov, S.D. Measurement and Calculation of Mechanical Properties of Silicone Rubber. Rus. Phys. J. 2021, 63, 1525-1529. [CrossRef]

8. Ahmed, K.; Nizami, S.S.; Raza, N.Z.; Shirin, K. Cure Characteristics, mechanical and swelling properties of marble sludge filled EPDM modified chloroprene rubber blends. Adv. Mater. Phys. Chem. 2012, 2, 90-97. [CrossRef]

9. Bunoiu, M.; Vlase, G.; Bica, I.; Balasoiu, M.; Pascu, G.; Vlase, T. Study of thermal stability of some magnetorheological elastomers. Rom. Rep. Phys. 2021, 73, 503.

10. Kruželák, J.; Hudec, I.; Dosoudil, R. Influence of thermo-oxidative and ozone ageing on the properties of elastomeric magnetic composites. Polym. Degrad. Stab. 2012, 97, 921-928. [CrossRef]

11. Zhao, G.; Shi, L.; Zhang, D.; Feng, X.; Yuan, S.; Zhuo, J. Synergistic effect of nanobarite and carbon black fillers in natural rubber matrix. Mater. Des. 2012, 35, 847-853. [CrossRef]

12. Salim, M.S.; Ariawan, D.; Rasyid, M.F.A.; Taib, R.M.; Thirmizir, M.Z.A.; Ishak, Z.A.M. Accelerated weathering and water absorption behavior of kenaf fiber reinforced acrylic based polyester composites. Front. Mater. 2020, 7, 7. [CrossRef]

13. Tang, N.; Lv, Q.; Huang, W.; Lin, P.; Yan, C. Chemical and rheological evaluation of aging characteristics of terminal blend rubberized asphalt binder. Constr. Build. Mater. 2019, 205, 87-96. [CrossRef]

14. Verma, A.R.; Reddy, B.S. Accelerated ageing studies of silicon-rubber based polymeric insulators used for HV transmission lines science direct. Polym. Test. 2017, 62, 124-131. [CrossRef]

15. Aziz, S.A.A.; Mazlan, S.A.; Ismail, N.I.N.; Choi, S.B.; Nordin, N.A.; Mohamad, N. A comparative assessment of different dispersing aids in enhancing magnetorheological elastomer properties. Smart Mater. Struct. 2018, 27, 117002. [CrossRef]

16. Chen, L.; Gong, X.L.; Jiang, W.Q. Investigation on magnetorheological elastomers based on natural rubber. J. Mater. Sci. 2007, 42, 5483-5489. [CrossRef]

17. Feldman, D. Polymer weathering: Photo-oxidation. J. Polym. Environ. 2002, 10, 163-173. [CrossRef]

18. Masłowski, M.; Miedzianowska, J.; Strzelec, K. Reinforced, extruded, isotropic magnetic elastomer composites: Fabrication and properties. Adv. Polym. Technol. 2019, 2019, 1-11. [CrossRef]

19. Li, X.; Bai, T.; Li, Z.; Liu, L. Inluence of the temperature on the hyper-elastic mechanical behavior of carbon black illed natural rubbers. Mech. Mater. 2016, 95, 136-145. [CrossRef]

20. Zając, P.; Kaleta, J.; Lewandowski, D.; Gasperowicz, A. Isotropic magnetorheological elastomers with thermoplastic matrices: Structure, damping properties and testing. Smart Mater. Struct. 2010, 19, 0450147. [CrossRef]

21. Akil, H.M.; Cheng, L.W.; Ishak, Z.M.; Abu Bakar, A.; Rahman, M.A. Water absorption study on pultruded jute fibre reinforced unsaturated polyester composites. Compos. Sci. Technol. 2009, 69, 1942-1948. [CrossRef] 
22. Dhakal, H.; Zhang, Z.; Richardson, M. Effect of water absorption on the mechanical properties of hemp fibre reinforced unsaturated polyester composites. Compos. Sci. Technol. 2007, 67, 1674-1683. [CrossRef]

23. Gu, H. Degradation of glass fibre/polyester composites after ultraviolet radiation. Mater. Des. 2008, 29, 1476-1479. [CrossRef]

24. Jia, Z.; Li, X.; Zhao, Q. Effect of artificial weathering on surface properties of unsaturated polyester (UP) resin. Mater. Chem. Phys. 2010, 121, 193-197. [CrossRef]

25. Chen, L.; Gong, X.; Li, W. Microstructures and viscoelastic properties of anisotropic magnetorheological elastomers. Smart Mater. Struct. 2007, 16, 2645-2650. [CrossRef]

26. Kashi, S.; Varley, R.; De Souza, M.; Al-Assafi, S.; Di Pietro, A.; de Lavigne, C.; Fox, B. Mechanical, thermal, and morphological behavior of silicone rubber during accelerated aging. Polym. Technol. Eng. 2018, 57, 1687-1696. [CrossRef]

27. Aziz, S.A.A.; Mazlan, S.A.; Ubaidillah, U.; Mohamad, N.; Choi, S.-B.; Aziz, M.A.C.; Johari, M.A.F.; Homma, K. Thermal aging rheological behavior of magnetorheological elastomers based on silicone rubber. Int. J. Mol. Sci. 2020, 21, 9007. [CrossRef]

28. Shaik, M.G.; Karuppaiyan, V. Effect of ageing on the tracking characteristics of high-temperature vulcanized silicone rubber hybrid composites for high voltage insulation. Materials 2020, 13, 2242. [CrossRef]

29. Chakraborty, R.; Reddy, B.S. Studies on high temperature vulcanized silicone rubber insulators under arid climatic aging. IEEE Trans. Dielectr. Electr. Insul. 2017, 24, 1751-1760. [CrossRef]

30. Moon, B.; Lee, J.; Park, S.; Seok, C.-S. Study on the aging behavior of natural rubber/butadiene rubber (NR/BR) blends using a parallel spring model. Polymers 2018, 10, 658. [CrossRef] [PubMed]

31. Woo, C.S.; Choi, S.S.; Lee, S.B.; Kim, H.S. Useful lifetime prediction of rubber components using accelerated testing. IEEE Trans. Reliab. 2010, 59, 11-17. [CrossRef]

32. Wei, H.; Guo, L.; Zheng, J.; Huang, G.; Li, G. Efect of nanosilica-based immobile antioxidant on thermal oxidative degradation of SBR. RSC Adv. 2015, 5, 62788-62796. [CrossRef]

33. Liu, J.; Li, X.; Xu, L.; Zhang, P. Investigation of aging behavior and mechanism of nitrile-butadiene rubber (NBR) in the accelerated thermal aging environment. Polym. Test. 2016, 54, 59-66. [CrossRef]

34. Ravindran, A.; Kamaraj, M.; Vasanthmurali, N.; Meghavarshini, V.; Balachandran, M. Nanosilica reinforced EPDM silicone rubber blends: Experimental and theoretical evaluation of mechanical and solvent sorption properties. Mater. Today 2021, 46, 4381-4386. [CrossRef]

35. Iryani, A.; Masudi, A.; Rozafia, A.I.; Hartanto, D.; Santoso, M.; Nur, H.; Azami, M.S. Enhanced removal of soluble and insoluble dyes over hierarchical zeolites: Effect of synthesis condition. Inorganics 2020, 8, 52. [CrossRef]

36. Masudi, A.; Jusoh, N.; Jusoh, R.; Jaafar, N.; Jalil, A.; Firdausi, A.; Hartanto, D. Equidistant crystal distortion arrangement of copper doped magnetite for paracetamol degradation and optimization with response surface methodology (RSM). Mater. Chem. Phys. 2020, 250, 122995. [CrossRef]

37. Perales-Martínez, I.A.; Palacios-Pineda, L.M.; Sánchez, L.M.L.; Martínez-Romero, O.; Puente-Córdova, J.; Elías-Zúñiga, A. Enhancement of a magnetorheological PDMS elastomer with carbonyl iron particles. Polym. Test. 2016, 57, 78-86. [CrossRef]

38. Palacios-Pineda, L.M.; Perales-Martinez, I.A.; Lozano-Sanchez, L.M.; Martinez-Romero, O.; Segura, E.; Puente-Córdova, J.; Elías-Zúñiga, A. Experimental Investigation of the Magnetorheological behavior of PDMS elastomer reinforced with iron micro/nanoparticles. Ploymers 2017, 9, 696. [CrossRef]

39. De Souza Neto, F.N.S.; Araújo, O.A.; Guilherme, L.R.; Garg, V.; Oliveira, A.C.; De Souza, P.E.; Júnior, A.F.; De Souza, P.E.N. Particles that slide over the water surface: Synthesis and characterization of iron oxides particles coated with PDMS, with hydrophobic and magnetic properties. Mater. Chem. Phys. 2015, 162, 100-105. [CrossRef]

40. Model, C.M.M.; Soria-Hern, C.G.; Palacios-Pineda, L.M.; El, A. Investigation of the effect of carbonyl iron micro-particles on the mechanical and rheological properties of isotropic and anisotropic MREs. Polymers 2019, 11, 1705.

41. Hanoosh, W.S.; Abdelrazaq, E.M. Polydimethyl siloxane toughened epoxy resins: Tensile strength and dynamic mechanical analysis. Malays. Polym. J. 2009, 4, 52-61.

42. Boczkowska, A.; Awietjan, S.F.; Babski, K. Effect of the processing conditions on the microstructure of urethane magnetorheological elastomers. In Smart Structures and Materials: Active Materials Behavior and Mechanics; Armstrong, W.D., Ed.; SPIE Digital Library: San Diego, CA, USA, 2006; Volume 6170, p. 61700R.

43. Wang, Y.; Zhang, X.; Oh, J.E. Fabrication and properties of magnetorheological elastomers based on CR/ENR self-crosslinking blends. Smart Mater. Struct. 2015, 24, 95006. [CrossRef]

44. Wang, Y.; Hu, Y.; Deng, H.X. Magnetorheological elastomers based on isobutylene-isoprene rubber. Polym. Eng. Sci. 2006, 46, 264-268. [CrossRef] 